\title{
Nurses: A Voice to Lead: Health is a Human Right - Tshwane District Nurses
}

\author{
Bethabile Lovely Dolamo* \\ Department of Health Studies, University of South Africa, South Africa
}

Received: 紫 August 28, 2018; Published: 制 September 05, 2018

*Corresponding author: Bethabile Lovely Dolamo, Department of Health Studies, University of South Africa, South Africa

\begin{abstract}
The purpose of this paper was to brief nurses under Tshwane District on the International Council of Nurses (ICN) 2018 International Nurses' Day (IND) theme. The ICN toolkit was downloaded and a full exploration of the topic was embarked on and delivered to Tshwane District nurses on 11 May 2018. The paper applies the context under which nurses practise as well as Hildegard Peplau's interpersonal relations model. This paper is divided into four parts: health is a human right; access to health care; investment and economic growth, and policy to practice - practice to policy. At the end the author makes recommendations according to context.
\end{abstract}

\section{Introduction and Background}

The International Council of Nurses (ICN) holds that health is a human right and is at the forefront of advocating for access to health and nurses are the key to delivering it ICN Toolkit [1]. The human right to health means that everyone has the right to the highest attainable standard of physical and mental health, which includes access to all medical services, sanitation, adequate food, decent housing, healthy working conditions, and a clean environment (National Economic and Social Rights Initiative [NESRI] sa). The case of Life Esidimeni in South Africa (Gauteng province) is a close example of health as a human right 'denied'. The case of Esidimeni is an example of individuals and communities who suffered and still suffer due to lack of access and could not afford health care; of 144 patients who died presumably due to neglect and hunger while 20 patients are still missing and unaccounted for Nicholson.

The president of the ICN, Annette Kennedy states that the right to health applies to nurses as well. "We know that improved quality and safety for patients depends on positive working environments for staff. That means the right to a safe working environment, adequate remuneration, and access to resources, and education. We must add in this the right to be heard and have a voice in decision making and policy development implementation!" Kennedy in ICN Toolkit [2]. Nurses and midwives in South Africa, this call is directed to you. South Africans have a Constitution South Africa, 1996 [3] that protects all citizens. Chapter 2 of the Constitution contains the 'Bill of Rights', and chapter 10 is on 'Public Administration - basic values and principles governing public administration', put simply by the late Zola Skweyiya as 'Batho Pele Principles' South Africa, 1997 [4].

In the Department of Health Strategic Plan, 2014/15 - 2018/19 South Africa [5], organizational environment is highlighted which covers working conditions. This paper discusses health as a human right, access to health care, investment and economic growth and policy to practice - practice to policy - in relation to the growth model of change and the ICN Toolkit, 2018 theme. Growth and development models of nursing look at the four central concepts of human as an intrinsic potential to change; human environment interaction as linear and irreversible, affecting change when applied at the appropriate time; health as change toward maximum potential, and examples of nursing implication stimulate re-patterning Leddy et al. [6]. Hildegard Peplau's interpersonal relations model is the chosen model to guide this paper

\section{Health is a Human Right}

In 2017 Puras presented a report stating the right of everyone to the enjoyment of the highest attainable standards of physical and mental health to the Human Rights Council of the United Nations ICN Toolkit [1]. The Constitution of the Republic of South Africa Act, 108 of 1996, places the obligation on the State to progressively realize socio-economic rights, including access to health care (Schedule 4; Section 9; Section 27 and 28 on health issues in the Constitution). In nursing and health practice, socially challenged groups including intellectually, cognitive and psychosocial disabilities, face neglect, abuse and violence at the hands of health practitioners. Meeting 
the physical health needs of diverse consumers with serious mental illness (SMI) is impeded by organizational, environmental, and consumer-level barriers. According to the South African Nursing Council (SANC), nursing education in South Africa as a four-year programme (R425) includes psychiatric nursing as one of the modules SANC, [7], and these abuses can be eradicated by nurses as they are prepared to manage psychosocial disabilities and as 'the voice to lead'.

Establishing better care coordination networks, increasing mental health provider education on medical issues, and culturallytailoring health promotion programming provide plausible strategies for improving the physical health of this vulnerable population Jerel et al. [8]. Mental health policies and services are said to be in crisis; for example, Esidimeni in Gauteng province, and Tower Hospital in the Eastern Cape Timeslive [9]. Since most professional nurses are psychiatrically trained, nurses should make a bold political commitment and urgent policy response in resolving this crisis ICN Toolkit [1]. The third issue is failure to respect, protect and fulfil the right to health. This means treating clients as human beings in the terrain of personal, social, political and economic lives not only treating the pathology, the nursing process that permits the professional nurse to view the client in a holistic way (nursing care plans are bound to cover these). And the fundamental issue constraining ability to attain health for all is the lack of a people-centred approach to health.

The fundamental of nursing practice is 'caring' and the 'Nurse's Pledge of Service' reminds nurses just that: Nursing is a work of heart. Health is a human right focuses on social determinants of health as ICN IND2017 theme, 'Nurses: the voice to lead - achieving sustainable development goals,' Section 27 of the SA Constitution covers this area South Africa. This year's (2018) theme is building on last year's theme by now exploring access to health. The right to health should be used as a constructive tool for the health sector to provide the best care for individuals, communities and populations. In Peplau's model, the nurse is concerned with the health needs of sick and well individuals, groups, families and communities Leddy \& Pepper [6]. Therefore, nursing helps people to meet their present needs. There are six (6) core elements of an effective health system based on the right to health, namely health services, health workforce, health informatics, health financing, leadershipgovernance, and stewardship World Health Organisation [10].

\section{Access to Health Care}

"If we do not engage consumers, patients, and family members in the health care process, we will not be effective at eliminating inequalities and improving health for all" (Kalahn Taylor-Clark in ICN Toolkit. Peplau views a person as an organism that lives in an unstable equilibrium and life is the process of striving in the direction of stable equilibrium Leddy \& Pepper [6]. Nursing is the only health profession that attends to people's needs in their most vulnerable times. This cannot be stated better than as expressed by the "Batho Pele Principles" South Africa [3]. Nurses are the closest health professionals who attend to clients' needs. Access to health care is the key determinant in how well the health system meets the health needs of individuals and communities. Health as a human right is the cornerstone of universal health coverage and peoplecentred care, and access is central to it. According to Peplau Leddy et al. [6], health implies forward movement of personality and other ongoing human processes in the direction of creative, constructive, productive, personal, and community living.

This International Nurses Day defines access as 'the opportunity to reach and obtain appropriate health care services in situations of perceived need for care'. Access is about 'enabling people to make the right steps of being able to contact or obtain health care as their needs require and having these health care needs fulfilled ICN Toolkit [1]. As in 'Batho Pele Principles', access does not only mean affordability, but involves both the user and the health service provider South Africa [4]. According to Peplau Leddy et al. [6], the relationship between the nurse and the client, which takes place within the environment, is a critical aspect of the therapeutic process. The following concepts are associated with the client's expectation of the health service provider ICN Toolkit [1]

a. Approachability - transparency, information, screening, outreach

b. Acceptability - professional values, norms, culture, gender

c. Availability and accommodation - geographical location, accommodation, hours of opening, appointments

d. Affordability - direct cost, indirect cost, opportunity cost

e. Appropriateness - technical and interpersonal quality, adequacy, coordination, and continuity.

The user should have the ability to:

a. Perceive - perception of needs and desire for care

b. Seek

c. Reach

d. Pay

e. Engage.

\section{Awareness and Identifying Unmet Needs}

When health literacy is poor, there is a higher incidence of other chronic conditions, poorer health and diminished quality of life. For health services to be accessible, people need to be aware that these services exist, understand how they can be accessed, and have a desire to use them. In the SABC 2 TV series on Health Talk about Primary Health (28/04/2018), community members said that there was no need to go to the clinic because the nurse would refer you to the doctor/specialist anyway. Make your clients know that 
they need this care; provide the service to assist in empowering the user to identify health needs and access the appropriate health services. Awareness and identifying unmet needs means improving access to comprehensive quality health care services.

Access to health services means the timely use of personal health services to achieve the best health outcomes. It requires three distinct steps: gaining entry into the health care system through insurance coverage; accessing a location where the needed health care services are provided and finding a health provider whom the patient trusts and can communicate with (Healthy people sa). According to the ICN Toolkit [11], for services to be accessible the first step is knowing that clients need the health care. The second step is that health care services are provided in a way that assists in empowering the user to identify health needs and access the appropriate health service.

\section{Meeting Diverse Needs}

People do not engage or access health services because of lack of culturally appropriate health care services; racist or discriminatory behaviour by health care staff; unaffordable cost of seeking health care, and lack of ability to attend appointments. Acceptability of health services extends to more than just cultural awareness ICN Toolkit. Meeting diverse needs includes gender, religion and other social factors, such as male nurse-female patient and vice versa. Many of these disparities may be due, at least in part to problems that racial and ethnic minorities may have in accessing and effectively utilizing the health care system. A solid and growing body of research shows differences in utilization levels across racial and ethnic groups and in the nature of the care that minority groups receive (AHRQ sa). These examples are an indication supporting the growing view that the health care system is not culturally competent or responsive to the country's health needs of the increasingly diverse population to provide care based on non-discrimination. Meeting the physical health needs of diverse consumers with serious mental illness (SMI) is impeded by organizational, environmental, and consumer-level barriers. Establishing better care coordination networks, increasing mental health provider education on medical issues, and culturally-tailoring health promotion programming provide plausible strategies for improving the physical health of this vulnerable population Jerel et al. [8].

\section{Availability and Easy-to-Reach Services}

Access in terms of availability of health services is a critical component in health and wellbeing. People living in rural areas often have poorer general health than those living in metropolitan areas due to significant difference in the social determinants of health (ICN-IND2018). According to ruralhealthinfo (sa), access to health care is critical to good health, yet rural residents face a variety of access barriers. Ideally, residents should be able to conveniently and confidently access services such as primary care, dental care, behavioural health, emergency care and public health services. Both farm and rural dwellers experience low to poor access to health care in terms of affordability, proximity, and quality, compared with their nonfarm and urban counterparts.

Health care institutions should provide satellite clinics to those populations to make easy-to-reach services available. Accessibility of or the ability to reach services is also affected by utilization of accessible transport to reach health services. The National Department of Health introduced engineering primary health care to reach out to such communities. The programme is ongoing but some communities are not benefiting from it as well as those whose work demands affect their ability to access health services during hours of operation Dolamo [12]. A twenty-four-hour health service at primary health care level has its own advantages and more disadvantages hence the service is failing.

\section{Affordability of Care}

The South African National Department of Health offers a free service especially to children under five and pregnant women. The South African government is now piloting the national health insurance (NHI) to assist poor and rich communities to be able to access all health services00. The two main sources of out-of-pocket expenditure are curative care and pharmaceuticals. However, obtaining care also includes travel costs, time away from work, child care or other parental arrangements and these costs are exacerbated when care is sought after hours. Moreover, with the ever-rising cost and expense of medications, geriatric patients are finding it difficult to get pharmaceutics these days.

\section{Safe, Quality Care}

AHRQ (sa) identifies six domains of health care quality: safe which means avoiding harm to patients from the care that is intended to help them; effective by providing services based on scientific knowledge to all who could benefit; patient-centred as care that is respectful and responsive; timely by reducing waiting and sometimes harmful delays; efficient by avoiding waste of equipment, supplies, ideas, and energy; equitable care that does not vary in quality because of personal characteristics such as gender, ethnicity, geographical location, and socioeconomic status. The ICN Toolkit 2018 cites the following example of results from an enquiry in a hospital where a number of patients were dying: It was found that the basic elements of care were neglected. Medications were either delayed or not given; patients were left unwashed for up to a month, and patients feared to be in hospital.

The report concluded that the primary cause of this substandard care was a chronic shortage of staff, particularly the nursing staff'. Another major problem related to heavy workloads, poor culture, poor leadership and limited resources. Clearly, services that are unsafe and of low quality lead to diminished health outcomes and even harm. If individuals and communities only have access to poor quality services, this is a restriction of access to health care. Access is not just about availability and affordability but also whether it 
is acceptable and effective. Currently health care is categorized by more to know, more to manage, more to watch, more to do, and more people involved in doing it than any time before. The evidence of quality improvement through nursing relates to skillmix, culture, education, staffing levels, leadership, interdisciplinary care, research, and informatics.

\section{Timeliness of Access}

Much of people's time is spent in a clinic waiting room. People find it very frustrating especially when accompanying a critically ill patient, because they cannot jump the line or stand up and go into a consulting room. It is even worse for health professionals and all these things are happening to them and they are aware of what is happening. Think of a patient with less than three weeks to live and a relative accompanying this patient. Instead of spending more time together at home (because of hope), they are made to be wait for hours in agony, tired, and emotionally unable to cope. Long waiting times may cause frustration, inconvenience, suffering and dissatisfaction with the health care system. Patients wake up very early in the morning to be at the front of the line, take lunch packs along, and still come back home late.

Long waiting times are barriers to accessing services, and a major source of anxiety and distress to patients and their support ICN Toolkit [1]. Health institutions and health professionals need to strategise how to minimize long waiting times for patients and clients to access services on time. In many instances, the earlier the treatment, the better the health outcome. Long waiting times potentially worsen symptoms, deteriorate the patient's condition, and lead to bad clinical outcomes. Registered nurses across the continuum of care are all critical contributors to preventing hospital admissions, improving waiting times, and reducing inefficiencies thereby creating accessible health services ICN Toolkit [1].

\section{People-Centred Care}

Nursing helps people to meet their present needs. When these needs are met, more mature needs can emerge and the person moves forward Leddy et al. [6]. "The client, not the nurse is the authority figure and decision maker, the nurse's role involves helping individuals and families in choosing the responsibilities for changing the health process' Rose Marie Risso Parse in ICN Toolkit [1]. As the nurses slip into the routine or delve into an overcrowded workplace, they lose awareness of the person as a whole, and lose sight of their uniqueness. Pattern-centred care refers to completing numerous tasks within a specific period of time with the risk that these tasks take precedence over engaging and meeting the holistic needs of the client. The common cause of pattern-centred care is the shortage of staff and skills-mix, and the caring component in nursing is lost in the process. Leverque, Harris and Grant [13] suggest that both resources and population characteristics can be modified to ensure continuing levels of access, but only resources can be modified in the short-term. Dimensions related to factors such as geographic access, resource availability, cultural acceptability, and quality of care to health system coverage have also been suggested (Equityhealth, sa).

\section{Investment and Economic Growth}

Owners of businesses usually focus on productivity without investing in human resources. Yet, happy and healthy people in the workplace are more productive than tired and unhealthy workers. Sen ICN Toolkit, [1] maintains that economic growth without investment in human development is unsustainable - and unethical. Health is a good investment - health as a human right with health as the backbone of the economy: no health no work, no wealth. A healthy nation is a productive nation. 'Healthy nurse, healthy nation' inspires nurses to use their skills as advocates, educators, and role models to improve the health of the nation (ANA, sa). Creating a healthy workplace environment for employees improves productivity and reduces costs related to absenteeism, turnover, workers' compensation and medical claims.

A healthy working environment is also important to keep the working community healthy for productivity. Consider Sibanye gold mine, with rock falling and stoppage of work with lack of productivity, and six dead mine workers in just one-month Cape talk [12]. Part of a healthy workplace environment is workplace culture - a great company culture can keep employees productive and even help the company retain the best employees, and happy workers are also more likely to stay (Inside, sa). It also involves physical, environmental and occupational health and safety, and health and lifestyle practice where employees care for the company they work for if they know that they are being looked after, including a supportive work environment. Dedicated nurses deserve the best practice environment, and to be supported to live the healthiest lives (ANA, sa). The challenge becomes the harmonization of health and economic policies to improve health outcomes whilst minimizing the negative effects on others.

The Aljeezira news Friday 04/05/2018, 11 h00 discussion was on contaminated water in Indonesia: The mother in the house was complaining that sometimes the water becomes black or red and she has to use it for the household, e.g. washing vegetables, cooking and all. The young boy complained that after showering with this water his skin became itchy and hot, and he also developed chest problems. The farmer had noticed that this water which he used for irrigation destroyed and reduced the production. His hands had also been affected by using this water. The observer in the situation reported that the introduction and development of factories in the area had brought this problem and the whole community was affected.

The health and wellness of people should be the central dimension of all policies because the health of the population underpins all other sectors of the economy. There are three policy directives that enable health as a human right: universal health 
coverage; people-centred care, and human resources for health. Universal health coverage means people having access to quality health care without suffering financial hardship. It addresses the determinants of health; that is, affordable, available, with a competent workforce, and effective and efficient health system. People-centred care is enshrined in human rights. It supports dignity, non-discrimination, participation, empowerment, access, equity and a partnership of equals. 'You treat disease, you win, you lose. You treat a person, I guarantee you win - no matter the outcome' (Patch Adams in ICN Toolkit, 2018). Human resources for health are essential. 'A strong nursing sector is the necessary building block of a strong health sector.

Nurses must be encouraged to take significant leadership roles in health policy, planning, and provision' (CK Mishra, Secretary, Ministry of Health \& Family Welfare, Government of India in ICN Toolkit [1]. Investing in the nursing workforce will see major returns on investment. In analyses of hospital quality, cost controls and nursing care have taken place independently of each other. It is time for these to change. These discussions need to be interwoven because improving health outcomes and creating efficiencies in health requires effective utilization and investment in the nursing workforce. Leaders should plan and invest in the nursing workforce; develop and enable them to practise; transform and empower them and retain and optimize their service.

\section{Policy to Practice - Practice to Policy}

Mason [14] ICN Toolkit, [6] maintains that all action, even inaction, is political. Politics is, after all, only the exercise of influence over the allocation of scarce resources or the use of relationships and power to gain ascendancy among competing stakeholders to influence policy and the allocation of scarce resources. As nurses, we argue that when policy is made we do not 'have a seat at the table'. What we must understand is that by the time the policy reaches the consultation 'table', the policy making is virtually complete. Where we need to be therefore is in the earliest stage of problem identification and solution framing. It is here that real influence happens [15-20]. Nurses must get more deeply engaged in understanding influence in policy making. Nurses need to know what is going on in the environment at this moment and how that might influence any policy conversation. Picking your time by assessing the context is critical to success in influencing policy. Knowing the participants enables you to choose who can help you to make a coalition with and whose influence needs to be countered and why.

The American Nursing Association (ANA) is committed to advancing nursing at every level, from policy that affects nurses to the working practice environment. By speaking through a unified voice on policy issues, and supporting nurses to practise to their full licence, education, and training; improving health care for all. Nurses advocate for their patients every day, but there are many opportunities for nurses to have their voices heard at the highest levels of policy making; by empowering nurses to excel; championing nurse interests and staying informed and making their voices heard (ANA sa) [21-33]. Nurses can move from practice to policy or from issue to action. Policy making is making specific things happen or not happen. Practice is an organized way in which an individual or a group carries out a particular activity. Policy is a set of statements about how a particular goal is to be reached. Much policy-making is about controlling practice. It is based on an assumption that it is possible to change the way people work by starting from a written statement about what should be done and how it should be done (Connected, sa).

\section{Recommendations Following this Presentation}

The author makes the following recommendations specifically to Tshwane District Nurses as essential transformers of health and the health care system. Correct me if I am wrong, but this district is highly privileged and strategically placed close to the offices of the Minister of Health - National Department of Health; next to the South African Nursing Council and Democratic Nursing Organisation of South Africa head offices; higher institutions of learning (Universities and colleges) and major hospitals and clinics. Therefore, based on their terms of reference, this district can be a Continuing Professional Development provider of basic standards of nursing practice and the Batho Pele Principles in order for the nurses in this district to:

a. Advocate for patients/clients (being that voice to lead)

b. Make a bold political commitment and urgent policy responses in solving health problems

c. Treat patients/clients as human beings - not only treating the pathology

d. Provide a people-centred not pattern-centred (routine and finishing procedures) approach to care

e. Allow the client and not the nurse practitioner to be the authority figure and decision maker and assist the client to take responsibility.

\section{Conclusion}

The finger is pointing at 'you' and 'me' in providing "Health is a Human Right", whatever our position. It is high time nurses take the lead in the formulation and evaluation of policies related to health and health provision. We need to stop pointing a finger somewhere else and blaming someone else for our problems and those occurring in the workplace. Nurses can be part of solving them by being involved in policy formulation. The National Department of Health should give nurses the dignity they deserve and improve nurses' working conditions.

\section{Acknowledgement}

The author is thankful to the Tshwane District Nurses for the invitation to present this paper at the annual International Nurses' 
Day, 2018 and to the International Council of Nurses for the Toolkit, 2018.

\section{References}

1. Agency for Healthcare Research and Quality. Sa. Providing care to diverse populations.

2. (2018) Agency for Healthcare Research and Quality. Sa. Advancing excellence in health care.

3. (2018) Aljeezira. World news Friday at eleven.

4. American Nursing Association (ANA). Sa. Healthy Nurse Healthy Nation.

5. (2018) Cape talk. Sibanye gold : we need to understand why mine deaths have increased.

6. (2018) Connected. Sa. The relation between policy and practice.

7. (2014) Department of Health (DOH). Health Strategic Plan, Pretoria: Government Printers.

8. Dolamo BL (2015) Proposed ward-based primary health care service: the case of Masealama, Limpopo province, South Africa. Africa Journal for Physical, Health Education, Recreation and Dance 1: 234-243.

9. Jean-Frederic Levesque, Mark F Harris, Grant Russell (2013) Patientcentred access to health care: conceptualising access at the interface of health systems and populationsEquity Health 12: 18.

10. Gauteng Health. Sa. Gauteng Health terminates Life Healthcare Esidimeni contract.

11. Improve access to comprehensive, quality health care services. Healthy People. Sa. Access to health services.

12. Holahan J, Karpman M, Zuckerman S (2016) Health care access and affordability among low- and moderate-income insured and uninsured adults under the Affordability Care Act.

13. Inside. sa, Creating a healthy workplace environment for your employees.

14. (2018) International Council of Nurses (ICN). 2018. Health is a Human Right: Access, Investment and Economic Growth. International Nurses Day Resources and Evidence.

15. (2017) International Council of Nursing, International Nurses Day theme, 'Nurses: the voice to lead.

16. Jerel ME, Siantz E, Leopold JC (2013) Contours of usual care: meeting the medical needs of diverse people with serious mental illnesses. Journal of Health Care for the Poor and Underserved, Johns Hopkins University Press, USA, 24(4): 1552-1573.

\section{ISSN: 2574-1241}

DOI: $10.26717 / B J S T R .2018 .08 .001697$

Bethabile Lovely Dolamo. Biomed J Sci \& Tech Res

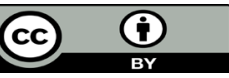

This work is licensed under Creative Commons Attribution 4.0 License

Submission Link: https://biomedres.us/submit-manuscript.php
17. Leddy S, Pepper JM (1993) Conceptual bases of professional nursing. ( $3^{\text {rd }}$ edn.). Lippincott, Philadelphia, USA.

18. Leverque JF, Harris MF, Russell G (2013) Patient-centred access to health care: conceptualizing access at the interface of health systems and populations. International Journal for Equity in Health 12: 18.

19. Mason (2016) in ICN Toolkit 2018. Nurses a voice to lead: health is a human right.

20. Mishra CK (2018) Secretary: Ministry of Health \& Family Welfare, Government of India, in International Council of Nurses (ICN). 2018. Health is a human right: access, investment and economic growth. International Nurses Day Resources and Evidence.

21. National Economic and Social Rights Initiative.

22. Nicolson G (2016) South Africa Life Esidimeni: Families of mentally ill patients blame MEC for deaths.

23. Rose Marie Risso Parse in ICN Toolkit. 2018. Nurses a voice to lead: health is a human right.

24. Rural Health Information. Sa. Health care access in rural communities.

25. SABC 2, 2018. TV series on Health Talk 28/04/2018, 10h00 -11h00 Primary health.

26. Sen A (2018) in ICN Toolkit. Nurses a voice to lead: health is a human right. ICN_Toolkit_2018_EN_Low Res South Africa. 1997. White Paper on transforming the Service Delivery "Batho Pele Principles". Pretoria: Government Printers.

27. (1994) South African Nursing Council. R425 (SANC 1994).

28. South Africa (Republic). 1996. Constitution of the Republic of South Africa, Act 108 of 1996. (Schedule 4; Section 9; Section 27 and 28). Pretoria: Government Printer.

29. Taylor-Clark K (2018) International Council of Nurses. 2018. Health is a Human Right: Access, Investment and Economic Growth. International Nurses Day Resources and Evidence.

30. The president of the ICN, Annette Kennedy

31. Timeslive (2018) Gauteng Health Department is corrupt and broke 16: 35.

32. (2010) World Health Organisation (WHO). Sa, Key components of a wellfunctioning health system 2 .

33. (2007) World Health Organisation (WHO) 2007. People-centred health care policy framework. WHO, Geneva, Switzerland.

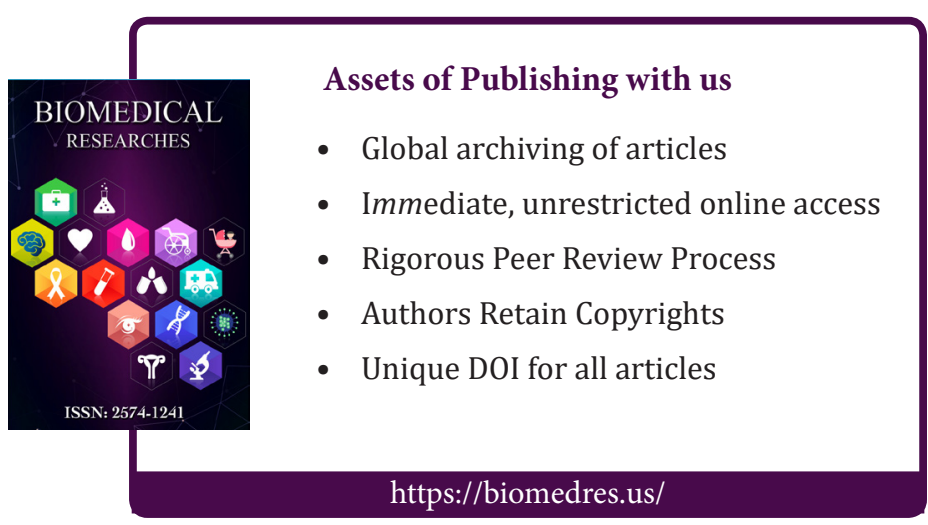

